\title{
Toward a Framework for Prototyping Physical Interfaces in Multiplayer Gaming: TwinSpace Experiences
}

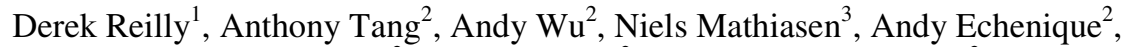 \\ Jonathan Massey $^{2}$, Hafez Rouzati ${ }^{2}$, and Shashank Chamoli ${ }^{2}$ \\ ${ }^{1}$ OCAD University, $100 \mathrm{McCaul}$ St. Toronto, ON, Canada \\ ${ }^{2}$ Georgia Tech GVU Center, 85 5th St. NW, Atlanta GA, USA \\ ${ }^{3}$ Aarhus University, Nordre Ringgade 1 DK-8000 Aarhus C Denmark \\ dreilly@faculty.ocad.ca, \{tonyt, andywu, aechenique, \\ ragingpotato, hafez,schamoli3\} agatech.edu, nielsm@cs.au.dk
}

\begin{abstract}
We reflect on our experiences using an experimental platform for rapidly prototyping physical control configurations for multiplayer games. We describe how the architecture permits novel forms of collaborative play through the combination and configuration of basic tangible/physical building blocks, the deep integration between physical and virtual objects, and flexibility in how physical and virtual spaces are mapped onto each other. We also identify three important limitations of the architecture that became apparent through our prototyping efforts.
\end{abstract}

\section{Introduction}

One of the main challenges in designing physical interfaces is the generation of usable prototypes (Greenberg \& Fitchett, 2001). This problem is especially pronounced in gaming, where physical interactions may need to be rapid, fluid, tightly synchronized, and readily interpreted by other players. Our goal is to build a platform to support the rapid design and implementation of novel physical interfaces for mixed-reality multiplayer games. We repurposed the TwinSpace framework for mixed-presence collaborative work (Reilly et al., 2010) as this platform to understand how elements of its design meet and fall short of this goal. We designed three games with this system: a shopping simulation, a scavenger hunt, and a mixed-reality card game. Due to space constraints, we describe only the scavenger hunt game. Our experiences revealed three problems that toolkits for prototyping games with physical interfaces need to address: first, the management of connection protocols and interconnections between components of the overall game; second, the translation layer between input/output components that may report/present information in different ways, and third, a common naming mechanism suitable for rapid prototyping. At the same time, our work validates the utility in this design space of three of TwinSpace's main features: its ability to deeply intertwine real and virtual objects, to define how real and virtual spaces are mapped onto each other, and to combine and configure heterogeneous collections of sensors and devices. 


\section{The Case for a Framework}

Infrastructure support for designing collaborative games involving physical interaction includes toolkits for designing physical interfaces, toolkits providing middleware support for confederated devices, and the content and game engines themselves. The basic problem with the current state of affairs is that these supports are disconnected. Input and middleware toolkits do not explicitly tie into content back-ends (e.g. an MMORPG engine), nor do they provide any support in specifying the mappings between the virtual world, the physical world, and the physical and virtual devices that inhabit these spaces.

Yet, game designers and researchers need to explore how to translate physical interactions into game actions, and how to manifest game events in the physical world. This calls for a software framework that is flexible and powerful enough to facilitate exploration of the many possible mappings between physical and virtual. Physical interfaces reflect and enforce collaborative gaming styles (Mandryk et al., 2002), as does the (relative) physical location of the players themselves (Benford et al., 2006). Because physical controls and mixed-presence configurations influence collaborative behaviour, a framework should support ways for researchers, designers and possibly gamers to define device configurations and real-virtual mappings.

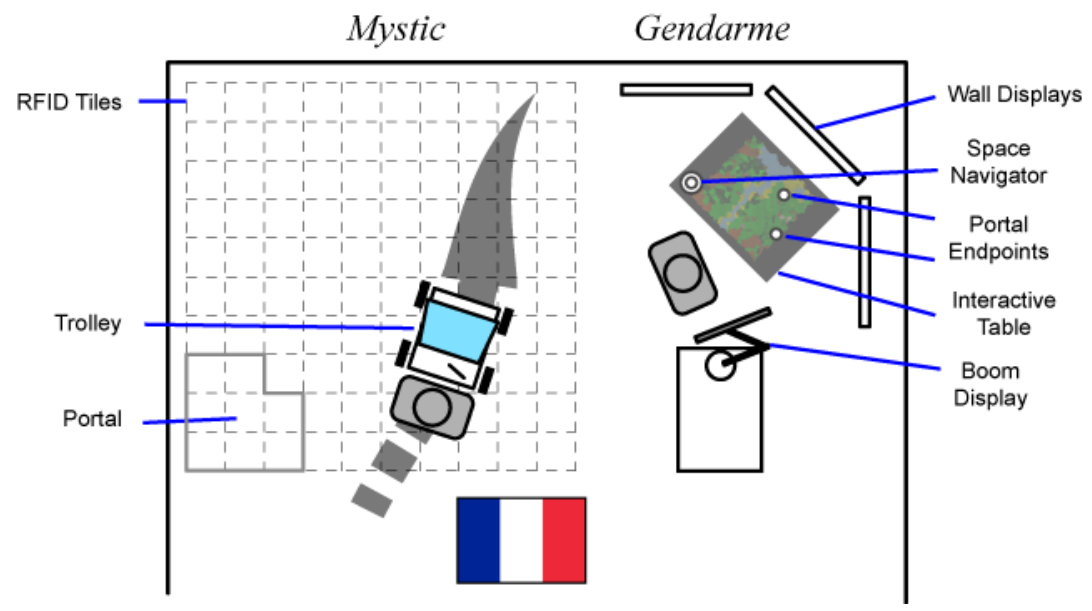

Fig. 1. Or de l'Acadie, French side. The Mystic collects riches using the trolley interface. The Gendarme seeks out desireable locations and guides the Mystic using a set of physical interfaces.

We have built a generic framework for prototyping collaborative mixed reality environments, including games, called TwinSpace (Reilly et al. (2010)). TwinSpace offers a range of methods for defining how a physical interface operates, in terms of the game space, the physical environment, and its relation to other game interfaces. One way this is achieved is by deeply interconnecting real and virtual spaces. For example, events generated by digital infrastructure in a physical space-such as sensor or input device events - can be published and reported in the virtual space. Likewise, events in the virtual space — such as an avatar entering a region, or a new 
player joining a game — can be published in the physical world. Further, TwinSpace offers facilities to define spatial and structural correspondence between physical and virtual spaces (for example, regions of a physical room can be mapped to regions in the virtual space). Finally, TwinSpace permits the definition and connection of heterogeneous clients (basic first-person mouse+keyboard clients, kiosks, instrumented spaces, etc.) to a shared game environment.

\section{Using the Framework: Benefits and Limitations}

In order to discuss our experiences, we briefly describe one of our game prototypes here. Or de l'Acadie is a game set in an abandoned $18^{\text {th }}$ Century French colonial fort. Two pairs (French and British sides) compete for valuables distributed throughout the fort, with the goal to collect the most valuables.

Each British player controls a single Soldier using keyboard controls. Players are not collocated, and can communicate with each other only when their soldiers are within earshot. Each Soldier is equipped with a static top-down map of the fort, and can collect a limited number of riches before needing to return to a location at the edge of the fort to stash them.

The French side consists of a Mi'kmaq mystic (the "Mystic"), and a deceased French soldier (the "Gendarme"). The French team is physically collocated (Figure 1 ), and uses a range of physical controls to locate and collect the valuables. While the 'living' players (French and British) are constrained by the laws of physics, the Gendarme moves freely through walls and in the air, but is unable to collect valuables. Instead, he communicates with the Mystic who collects them.

The Mystic pushes a cart, and does not need to periodically stow the riches like the British Soldiers do, but as a result he cannot move quickly, or climb stairs. Luckily, the Gendarme can transport the Mystic to any location in the fort by creating a portal between the Mystic's current location and the desired destination. The game is described in more detail in Reilly et al. (2010).

Mixed reality games combine virtual and physical to create a fused game space. This asymmetry between the worlds and players' experiences of them provides interesting design opportunities. For example, interfaces can be tailored to promote advantageous multi-role strategies of gameplay. The flexibility of TwinSpace proved invaluable during our design process: by allowing us to easily connect, and re-map physical interfaces with game entities, it gave us the ability to explore novel combinations and mappings rapidly.

The framework allowed us to move quickly between quite different overall game designs for Or de l'Acadie. Three key design changes were made that impacted the game scenario. The first was to define two players for the French side: the Gendarme, whose interface consisted of the table, wall displays and SpaceNavigator, and the Mystic, whose interface consisted of the trolley. TwinSpace allowed us to decouple the trolley from the immersive view very easily. The second was to provide a single interface for controlling the Gendarme's position (the SpaceNavigator), using the tabletop only as a dynamic view of the fort and as an interface for placing portal endpoints. Moving portal endpoints sent a message to the game server to reconfigure the in-game portal accordingly. Finally, as an all-seeing ghost, we allowed the Gendarme to see what the Mystic sees, in order to help him locate riches. The existing 
report of position and orientation given by the trolley for the first design was quickly remapped to control the home position of the Gendarme's boom display in the final design. Rotating the display on the boom causes the orientation of the camera to change relative to the Mystic's perspective (accomplished using a MotionNode sensor). Similarly, the existing report of position and orientation given by the trolley was remapped to control the home position of the Gendarme's boom display.

When using the framework we also identified three required improvements:

Timely and Appropriate Communication between Components. For example, our games made use of a moderate number of sensors ( 10), but even so we would sometimes experience synchronization or message latency issues. While generally imperceptible with a small number of interfaces, scalability was an issue.

Translation Layers between Interconnected Components. When combining components in novel ways, we frequently encountered format mismatches. While TwinSpace currently provides some mapping capacity to apply basic transforms, it was sometimes not sophisticated enough.

Consistent Naming of Entities. During prototyping quick hacks were often written that hard-coded device or virtual object IDs to test quickly, because the mapping component did not provide an interface to define IDs globally or to query object IDs based on desired attributes.

\section{Conclusion}

TwinSpace provides key features that facilitate the development of collaborative gaming prototypes employing physical interfaces. By providing many options for connectivity between physical and virtual while simultaneously centralizing mapping details, and by supporting heterogeneous gaming interfaces, TwinSpace allowed us to specify and reconfigure interfaces and gaming scenarios as a team in an iterative, exploratory fashion. Our prototypes have combined multiple interface elements, yielding interfaces tailored for unique forms of collaborative gameplay. Our experiences also highlight some important current limitations of the framework that will need to be addressed in order to provide more complete support for these prototyping activities.

Acknowledgments. This work was funded by NSF grant IIS-0705569, and a research collaboration grant from Steelcase Inc.

\section{References}

1. Benford, S., Crabtree, A., Flintham, M., Drozd, A., Anastasi, R., Paxton, M., Tandavanitj, N., Adams, M., Row-Farr, J.: Can you see me now? TOCHI 13(1), 100-133 (2006)

2. Greenberg, S., Fitchett, C.: Phidgets: easy development of physical interfaces through physical widgets. Proc UIST 2001, 209-218 (2001)

3. Mandryk, R.L., Maranan, D.S.: False prophets: exploring hybrid board/video games. In: Ext. Abstracts of CHI 2002, pp. 640-641 (2002)

4. Reilly, D., Rouzati, H., Wu, A., Hwang, J.Y., Brudvik, J., Edwards, W.K.: TwinSpace: a Framework for Cross-Reality Teamspaces. In: Proc. UIST 2010 (2010) 